\title{
Algebraic Localization from Power-Law Couplings in Disordered Quantum Wires
}

\author{
Thomas Botzung, ${ }^{1,2}$ Davide Vodola,, , | Piero Naldesi, ${ }^{4}$ Markus Müller, ${ }^{3}$ Elisa Ercolessi, ${ }^{2,5}$ and Guido Pupillo ${ }^{1, f}$ \\ ${ }^{1}$ University of Strasbourg, CNRS, ISIS (UMR 7006) and IPCMS (UMR 7504), 67000 Strasbourg, France \\ ${ }^{2}$ Dipartimento di Fisica e Astronomia dell'Università di Bologna, I-40127 Bologna, Italy \\ ${ }^{3}$ Department of Physics, Swansea University, Singleton Park, Swansea SA2 8PP, United Kingdom \\ ${ }^{4}$ Université Grenoble-Alpes, LPMMC, F-38000 Grenoble, France \\ and CNRS, LPMMC, F-38000 Grenoble, France \\ ${ }^{5}$ INFN, Sezione di Bologna, I-40127 Bologna, Italy
}

\begin{abstract}
We analyze the effects of disorder on the correlation functions of one-dimensional quantum models of fermions and spins with long-range interactions that decay with distance $\ell$ as a power-law $1 / \ell^{\alpha}$. Using a combination of analytical and numerical results, we demonstrate that power-law interactions imply a longdistance algebraic decay of correlations within disordered-localized phases, for all exponents $\alpha$. The exponent of algebraic decay depends only on $\alpha$, and not, e.g., on the strength of disorder. We find a similar algebraic localization for wave-functions. These results are in contrast to expectations from short-range models and are of direct relevance for a variety of quantum mechanical systems in atomic, molecular and solid-state physics.
\end{abstract}

\section{INTRODUCTION}

Quantum waves are generally localized exponentially by disorder. Following the seminal work by Anderson with spinpolarized electrons ${ }^{1}$ much experimental $\sqrt{2}$ and theoretical interest has been devoted to the study of localized phases and to the localization-delocalization transition for non-interacting and interacting quantum models 9 , 30 .

While most works have focused on short-range couplings, long-range hopping and interactions that decay with distance $\ell$ as a power-law $1 / \ell^{\alpha}$ have recently attracted significant interes $[31-45$ as they provide novel physical effects and can be now engineered in a variety of atomic, molecular and optical systems. For example, the Ising model with powerlaw spin interactions with tunable exponent $0<\alpha<3$ has been first realized in breakthrough experiments with arrays of laser-driven cold ions 56 and could also be obtained with atoms trapped in a photonic crystal waveguide $51-54$; dipolartype $1 / \ell^{3}$ or van-der-Waals-type $1 / \ell^{6}$ couplings have been recently demonstrated experimentally with ground-state neutral atoms 55 -58, Rydberg atoms 59 - 73 , polar molecules $74-76$ and nuclear spins 77 . In solid state materials, power-law hopping is of interest for, e.g., excitonic materials 78 ; ; long-range $1 / \ell$ coupling is found in helical Shiba chains 9192 , made of magnetic impurities on an s-wave superconductor, while planar arrays of Josephson Junctions ${ }^{93 / 94}$ can effectively realize longrange extensions of the Kitaev chain for spinless fermions 95 . In the absence of disorder, theory and experiments have provided evidence for novel enticing static and dynamic phenomena in these systems, such as, e.g., the non-local propagation of correlations 33 , time crystal phases 97 , novel topological effects 103 , and exotic behaviors of equal-time correlations, such as hybrid exponential and power-law decays within gapped phases, related to the violation of the area law for the entanglement entropy 104 107. However, in many of these systems, disorder - in particles' positions, local energies, or coupling strengths - is an intrinsic feature. Understanding its effects on the above phenomena and in the context of singleparticle and many-body localization remains a fundamental open question.
For non-interacting models, it is generally expected that long-range hopping induces delocalization in the presence of disorder for $\alpha<d$, while for $\alpha>d$ all wave-functions are exponentially localized $1 / 08-114$. However, recent theoretical works with positional ${ }^{115}$ and diagonal 114 disorder have demonstrated that localization can survive even for $\alpha<$ $d$. Surprisingly, wave-functions were found to be localized only algebraically in these models, in contrast to the usual Anderson-type exponential localization expected from shortrange models. How these finding translate to the behavior of wave-functions and, crucially, correlation functions in manyparticle systems is not known.

In this work, we investigate the effects of disorder on the decay of correlation functions and wave-functions in long-range quantum wires of fermions and spins. These are extensions of the Kitaev chain with long-range pairing $93|104| 116$ and the Ising model in a transverse field 117 , providing both analytical insight and immediate experimental interest. While in the absence of disorder and for short-range interactions they are identical, for long-range interactions they correspond to integrable and non-integrable chains, respectively. For fermions, we determine the regimes of localization for all $\alpha$ for the cases of disordered hopping or pairing. For the Ising chain, we focus on the regime $\alpha>1$, where the disordered phase diagram has been shown to display many-body localization theoretically ${ }^{118}$ and experimentally ${ }^{7}$. For all models we compute the one-body and two-body connected correlation functions, finding several novel features: (i) The connected correlation functions decay algebraically at long distance within all localized phases, (ii) with an exponent that depends exclusively on $\alpha$, and not, e.g., on the disorder strength. (iii) For the fermionic models, we derive analytic results for the long-distance decay of the correlations that explain the found algebraic decay, in excellent agreement with the numerics. (iv) The same analytical predictions are found to hold also for the correlations of the interacting Ising chain. (v) For any $\alpha$, the localized wavefunctions of the fermionic models display a long-distance algebraic decay with exponent $\alpha$, different from recent predictions for long-range hopping models. These results should be of direct relevance to many experiments in cold atomic, molecular and solid-state physics with fermions and spins. 


\section{THE MODELS}

We consider the following Hamiltonians for onedimensional long-range fermionic models

$$
H_{\mathrm{I}, \mathrm{II}}=H_{0}+V_{\mathrm{I}, \mathrm{II}}
$$

where $H_{0}$ is a homogeneous Hamiltonian given by

$$
\begin{aligned}
H_{0} & =-t \sum_{j=1}^{L}\left(a_{j}^{\dagger} a_{j+1}+\text { H.c. }\right)+\mu \sum_{j=1}^{L} n_{j} \\
& +\sum_{j, \ell} \frac{\Delta}{\ell^{\alpha}}\left(a_{j} a_{j+\ell}+\text { H.c. }\right)
\end{aligned}
$$

that describes a $p$-wave superconductor with a long-range pairing, and the indices I, II refer to the two different types of Hamiltonians we consider, namely

$$
V_{\mathrm{I}}=\sum_{j=1}^{L} W_{j}\left(a_{j}^{\dagger} a_{j+1}+\text { H.c. }\right)
$$

that corresponds to a random hopping and

$$
V_{\mathrm{II}}=\sum_{j, \ell} \frac{W_{j}}{\ell^{\alpha}}\left(a_{j} a_{j+\ell}+\text { H.c. }\right)
$$

that corresponds to a random long-range pairing. In the previous equations, $a_{j}^{\dagger}\left(a_{j}\right)$ is a fermionic creation (annihilation) operator on site $j, \mu$ is the chemical potential, $n_{j}=a_{j}^{\dagger} a_{j}$ and $W_{j}$ are i.i.d random variables drawn from a uniform distribution of width $2 W$ and zero mean value. We fix the energy scale by letting $\Delta=2 t=1$ and we choose $\mu=2.5$, corresponding to a gapped paramagnetic phase ${ }^{104}$ for $W_{j}=0$. Different values of $\mu$ do not change the results we find in the following. The random Hamiltonians (1) can be written in diagonal form as $H_{\mathrm{I}, \mathrm{II}}=\sum_{q=0}^{L-1} \Lambda_{q} \eta_{q}^{\dagger} \eta_{q}$ by a generalized Bogoliubov transformation 119 defined by $\eta_{q}=\sum_{j}\left(g_{q, j} a_{j}+h_{q, j} a_{j}^{\dagger}\right)$, with $\Lambda_{q}$ the energies of the single-particle states labelled by $q$. The ground state $|\Omega\rangle$ is then the vacuum of all quasi-particles $\eta_{q}$ and the matrix elements $g_{q, j}$ and $h_{q, j}$ can be identified with the wave functions of the two fermionic modes $\eta_{q}^{\dagger}$ and $\eta_{q}$, respectively.

As an interacting model, we consider the following random long-range Ising Hamiltonian ${ }^{117}$ in transverse field

$$
H_{\mathrm{LRI}}=\sum_{j, \ell}\left(\sin \theta+B_{j, j+\ell}\right) \frac{\sigma_{j}^{x} \sigma_{j+\ell}^{x}}{\ell^{\alpha}}+\sum_{j=1}^{L}\left(\cos \theta+W_{j}\right) \sigma_{j}^{z}
$$

where $\sigma_{j}^{v}(v=x, z)$ are Pauli matrices for a spin-1/2 at site $j$ and $B_{j, j+\ell}$ are i.i.d random variables drawn from a uniform distribution of width $2 B$ and zero mean value. We choose $\theta=\pi / 5$, corresponding to a paramagnetic phase ${ }^{116}$ for $B_{j, j+\ell}=W_{j}=0$. Different values of $\theta$ will not change the results we find in the following. For any finite disorder strength, the model Eq. (5) displays a many-body localized (MBL) phase 120 for $\alpha>1$.

In the following, we first determine the regimes of localization for the fermionic models Eqs. (1) and then compute
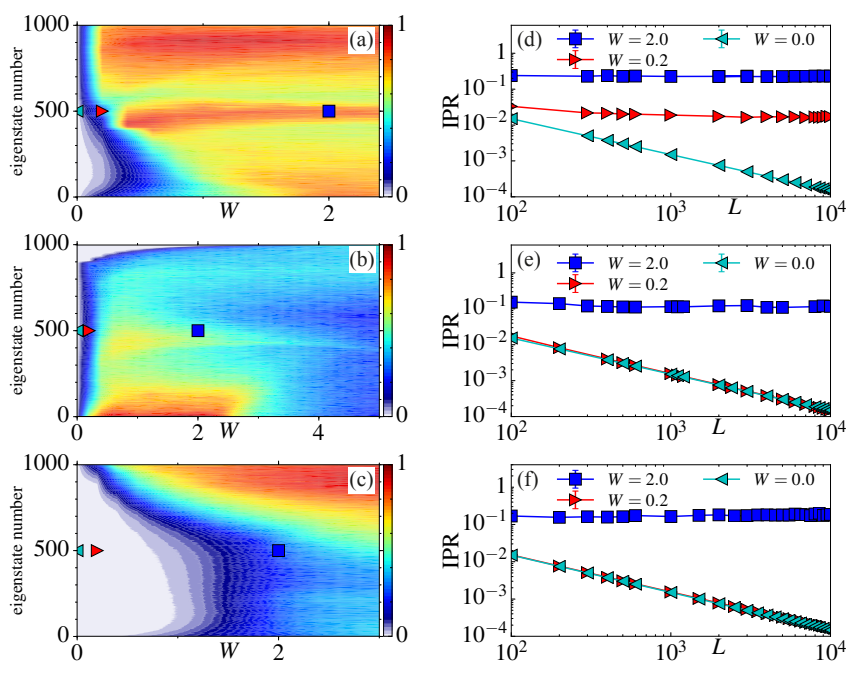

FIG. 1. Left panels: IPR for a system of $L=1000$ sites as a function of the disorder strength $W$ for (a) $\alpha=3$ and (b) $\alpha=0.8$ for the model (I) with random hopping and (c) for $\alpha=3.0$ for the model (II) with random pairing. Only in these panels, for drawing purpose, the IPR has been rescaled to 1 in correspondence of its maximum value. Right panels: Scaling of the IPR for the states at the centre of the band as a function of the system size $L$ and different $W$ for (d) $\alpha=3$ and (e) $\alpha=0.8$ for the model (I) and (f) for $\alpha=3.0$ for the model (II). In panels (a-c) the symbols indicate the values of $W$ that we choose to plot the IPR in panels (d-f).

the single and the two-body correlation functions, as well as the wave-functions, within the localized phases using analytical and numerical techniques. For the long-range Ising model Eq. (5) we compute the spin-spin connected correlation functions numerically. Our goal is to demonstrate that all these quantities decay algebraically at large distances both for noninteracting and interacting MBL localized models and to characterize their decay exponents.

\section{LOCALIZED PHASES OF DISORDERED FERMIONS}

We determine the localized phases for Hamiltonians Eqs. (1) by combining information from the inverse participation ratio (IPR). The IPR gives information about the spatial extension of single-particle states and is defined as $\mathrm{IPR}_{q}=$ $\sum_{j=1}^{L}\left[\left|g_{q, j}\right|^{4}+\left|h_{q, j}\right|^{4}\right]$ for a normalized state with energy $\Lambda_{q}$. The IPR tends to zero for increasing $L$ for extended states, while it remains finite for localized states.

Figure 1 shows the IPR as a function of $W$ for all the single particle eigenstates of a system of $L=1000$ for model Eqs. (1) (I) [for $\alpha=3$ and 0.8 in panels (a) and (b), respectively], and (II) [for $\alpha=3$ in panel (c)] together with examples of finite size scaling [panels (d-e)].

For model (I) with disordered hopping and $\alpha>1$ [panel (a)] essentially all states are localized. For $\alpha<1$ [panel (b)] we find that, at $W$ fixed, there exists an energy below (above) which all the states are localized (delocalized). However, the fraction of delocalized states that are found at high energy 
scales to zero as $\sim 1 / L^{1 /(4-2 \alpha)}$ and those states approach the band edge for $L \rightarrow \infty$ [see Appendix B]. For model (II) with disordered pairing when $\alpha>1$ localized states are present at all energies if $W \gtrsim 2$ [panel (c)].

\section{CORRELATION FUNCTIONS}

We consider the single-particle correlator $C(j, \ell)=$ $\left\langle a_{j}^{\dagger} a_{j+\ell}\right\rangle_{W}$ for the two free-fermionic models of Eqs. (1) as well as the spin-spin correlation function $S_{v}(j, \ell)=$ $\left[\left\langle\sigma_{j}^{v} \sigma_{j+\ell}^{v}\right\rangle-\left\langle\sigma_{j}^{v}\right\rangle\left\langle\sigma_{j+\ell}^{v}\right\rangle\right]_{W}$ (for $v=x, z$ ) for the interacting long-range Ising model of Eq. (5) in the localized phases. In the definitions of $C(j, \ell)$ and $S_{v}(j, \ell)$ the subscript $W$ indicates averaging over the disorder distribution. For models with short-range interactions, all the correlation functions decay exponentially with $\ell$. For models with-long range coupling, it has been shown that correlation functions can display a power-tail at long distance [see Appendix A]. Here we are interested in the effects that can originate from the interplay between long-range interactions and disorder.

Figures 2(a) and (b) show the correlator $C(\ell):=C\left(j_{0}, \ell\right)$ for models $H_{\mathrm{I}}$ and $H_{\mathrm{II}}$, respectively, for different values of $\alpha$. We choose $j_{0}=L / 4$ far from the edges in order to avoid boundary effects. We find numerically that the long-distance decay of $C(\ell)$ is always of power-law type $C(\ell) \sim \ell^{-\gamma}$ for all $\alpha$ within localized phases. In particular, for model $H_{\mathrm{I}}$ [panel (a)] and $\alpha<1$ the decay is essentially algebraic at all distances with $\gamma \sim 2-\alpha$, while for $\alpha>1$ we find for both models a hybrid decay that is exponential at short distances and power-law at large distances, with $\gamma \sim \alpha$ [panels (a) and (b)]. Surprisingly, we find that the values of the decay exponents of the power-law tails do not depend on the disorder strength [see Appendix C]. Remarkably, we observe that this behaviour is typical also of states far from the bottom of the energy band. This is shown in Appendix C, where we compute the onebody correlation function of a localized single particle state at the centre of the energy band. This is reminiscent of recent results for fermions at finite temperature, in the absence of disorder ${ }^{43}$.

This surprising long-distance behavior of correlations can be understood by computing the correlations analytically treating disorder as a perturbation. Here, we focus on model (I) with perturbation $V_{\mathrm{I}}$. The homogeneous Hamiltonian $H_{0}$ can be diagonalised via Fourier and Bogoliubov transformations as $H_{0}=\sum_{k} \lambda_{\alpha}(k) \xi_{k}^{\dagger} \xi_{k}$, where $\lambda_{\alpha}(k)=\left[(\cos k-\mu)^{2}+\right.$ $\left.4 f_{\alpha}^{2}(k)\right]^{1 / 2}$ and $\xi_{k}$ are extended Bogolioubov quasi-particles related to the unperturbed fermionic operators in momentum space via $\tilde{a}_{k}=v_{k} \xi_{k}-u_{k} \xi_{-k}^{\dagger}$ with $v_{k}=\cos \varphi(k)$ and $u_{k}=$ isin $\varphi(k)$, with $\tan 2 \varphi(k)=f_{\alpha}(k) /[\mu-\cos k]$ and $f_{\alpha}(k)=$ $\sum_{\ell=1}^{L-1} \sin (k \ell) / \ell^{\alpha}$. At first order in $W_{j}$ the ground state $\left|\Omega_{0}\right\rangle$ of the unperturbed Hamiltonian $H_{0}$ is modified by $V_{\mathrm{I}}$ as

$$
|\Omega\rangle=\left|\Omega_{0}\right\rangle+\left|\delta \Omega_{0}\right\rangle=\left|\Omega_{0}\right\rangle-\sum_{k k^{\prime}} J_{k, k^{\prime}} A\left(k, k^{\prime}\right) \xi_{k}^{\dagger} \xi_{k^{\prime}}^{\dagger}\left|\Omega_{0}\right\rangle,
$$

where we define $J_{k, k^{\prime}}=-\sum_{j} e^{\mathrm{i}\left(k-k^{\prime}\right) j} W_{j} / L$ and $A\left(k, k^{\prime}\right)=$ $2\left(\mathrm{e}^{\mathrm{i} k}+\mathrm{e}^{-\mathrm{i} k^{\prime}}\right) v_{k} u_{k^{\prime}}^{*} /\left[\lambda(k)+\lambda\left(k^{\prime}\right)\right]$. We find that $\langle\Omega|H| \Omega\rangle$ provides an excellent approximation of the exact ground-state energy for $0<W<3$ [see Appendix $\mathrm{C}$ ], suggesting that $|\Omega\rangle$ is a good approximate ground-state in that range. For correlations, since $\left\langle J_{k, k^{\prime}}\right\rangle_{W}=0$, we find that $\left\langle\delta \Omega_{0}\left|a_{j}^{\dagger} a_{j+\ell}\right| \Omega_{0}\right\rangle_{W}$ and $\left\langle\Omega_{0}\left|a_{j}^{\dagger} a_{j+\ell}\right| \delta \Omega_{0}\right\rangle_{W}$ vanish due to averaging over the disorder distribution. We obtain the following expression for $C(\ell)$

$$
\left\langle\Omega\left|a_{j}^{\dagger} a_{j+\ell}\right| \Omega\right\rangle_{W}=\left\langle\Omega_{0}\left|a_{j}^{\dagger} a_{j+\ell}\right| \Omega_{0}\right\rangle+\left\langle\delta \Omega_{0}\left|a_{j}^{\dagger} a_{j+\ell}\right| \delta \Omega_{0}\right\rangle_{W} .
$$

The first term in the r.h.s. of Eq. (7) corresponds to the correlator of the homogeneous system 116 that is $\left\langle\Omega_{0}\left|a_{j}^{\dagger} a_{j+\ell}\right| \Omega_{0}\right\rangle=\int_{0}^{2 \pi} \mathrm{d} k \mathrm{e}^{\mathrm{i} k \ell} R_{0}(k)$, with $R_{0}(k)=\left|u_{k}\right|^{2}$. The second term arises instead because of the random part of the Hamiltonian and reads

$$
\left\langle\delta \Omega_{0}\left|a_{j}^{\dagger} a_{j+\ell}\right| \delta \Omega_{0}\right\rangle_{W}=\frac{2 W^{2}}{3} \int_{0}^{2 \pi} \mathrm{d} k \mathrm{e}^{\mathrm{i} k \ell} R_{1}(k),
$$

where we have defined $R_{1}(k)=[c-U(k)]\left|u_{k}\right|^{2}-V(k)\left|v_{k}\right|^{2}$, with $c$ that does not depend on $k, V(k)=\sum_{p} A(p, k) A(k, p) \sim$ $f_{\alpha}(k) / \lambda_{\alpha}(k)$ and $U(k)=V(-k)$. The behaviour of both integrals for $\ell \rightarrow \infty$ can be extracted by integrating $R_{0}(k)$ and $R_{1}(k)$ for $k \rightarrow 0$. In this limit, $f_{\alpha}(k)$, and thus the single-particle energy $\lambda_{\alpha}(k)$, display a non-analytical scaling $f_{\alpha}(k) \sim|k|^{\alpha-1}$.For the first term in the r.h.s. of Eq. (7) the latter behavior results in (details in Appendix C)

$$
\left\langle\Omega_{0}\left|a_{j}^{\dagger} a_{j+\ell}\right| \Omega_{0}\right\rangle \sim \begin{cases}1 / \ell^{2-\alpha} & \text { for } \alpha<1 \\ 1 / \ell^{2 \alpha-1} & \text { for } 1<\alpha<2 \\ 1 / \ell^{\alpha+1} & \text { for } \alpha>2\end{cases}
$$

which corresponds to the expected long-distance power-law decay of correlation functions for the homogeneous gapped superconductor with long-range pairing $104 \mid 116 / 125 / 127]$. Instead, for $R_{1}(k)$ the scaling of $f_{\alpha}(k)$ near $k \rightarrow 0$ implies

$$
R_{1}(k) \sim \begin{cases}k^{1-\alpha} & \text { for } \alpha<1 \\ k^{\alpha-1} & \text { for } \alpha>1\end{cases}
$$

which entails the following form of the disordered part of $C(\ell)$

$$
\left\langle\delta \Omega_{0}\left|a_{j}^{\dagger} a_{j+\ell}\right| \delta \Omega_{0}\right\rangle_{W} \sim \begin{cases}W^{2} / \ell^{2-\alpha} & \text { for } \alpha<1 \\ W^{2} / \ell^{\alpha} & \text { for } \alpha>1\end{cases}
$$

after the integration of $R_{1}(k)$ in Eq. (8)

The discussion above demonstrates the following surprising results: (i) For $\alpha<1$ disorder does not modify the power of the algebraic decay of correlations, rather it affects its strength. (ii) For $\alpha>1$, the decay of correlations due to disorder is always algebraic, with an exponent that is smaller than for the homogeneous case with $W_{j}=0$. This implies that disorder enhances algebraic localization in these gapped models. (iii) For $\alpha \leq 2$ we find the duality relation $\gamma(\alpha)=\gamma(2-\alpha)$ in the exponents of the algebraic decay. This is reminiscent of the duality recently found for the decay exponent of the wave functions of long-range non-interacting spin models with positional disorder ${ }^{115}$. We come back to this point below. 

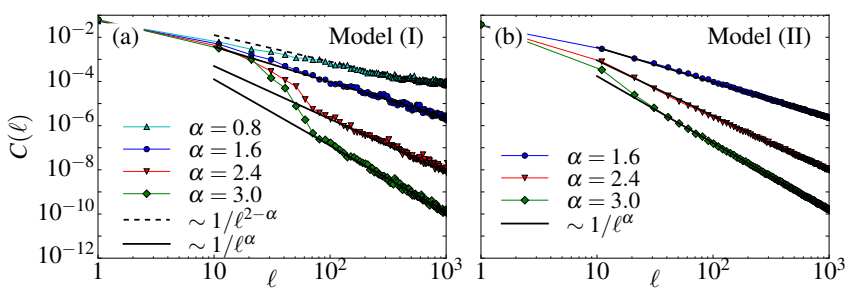

FIG. 2. (a) Correlation function $C(\ell)$ for the model (I) as a function of the lattice site $\ell$ for different values of $\alpha$ and for $W=5, L=2000$ and 400 disorder realizations. The power-law tails are fit by the black lines scaling as $1 / \ell^{2-\alpha}$ (dashed) and $1 / \ell^{\alpha}$ (solid) in agreement with the analytical results in Eq. (11). (b) Same as panel (a) but for the model (II).

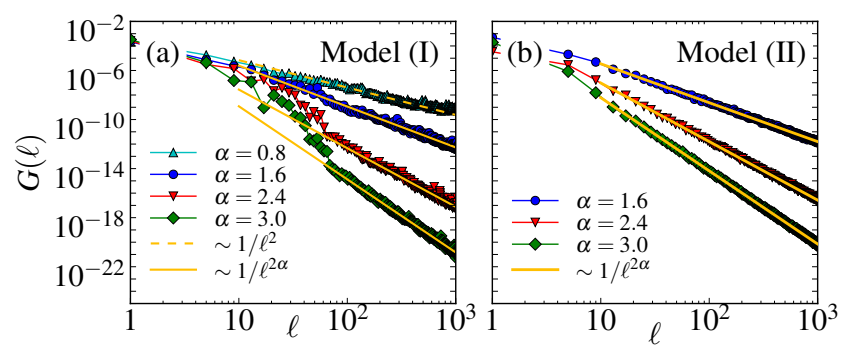

FIG. 3. (a) Density-density correlation function $G(\ell)$ for the model (I) as a function of the lattice site $\ell$ for different values of $\alpha$ and for $W=5, L=2000$ and 400 disorder realizations. The powerlaw tails are fit by the yellow lines scaling as $1 / \ell^{2}$ (dashed) and $1 / \ell^{2 \alpha}$ (solid). (b) Same as panel (a) but for the model (II).

From the single-particle correlators $\left\langle a_{j}^{\dagger} a_{j+\ell}\right\rangle$ and $\left\langle a_{j}^{\dagger} a_{j+\ell}^{\dagger}\right\rangle$, by means of the Wick theorem, we computed also the densitydensity correlation functions

$$
\begin{aligned}
G(j, \ell) & =\left[\left\langle n_{j} n_{j+\ell}\right\rangle-\left\langle n_{j}\right\rangle\left\langle n_{j+\ell}\right\rangle\right]_{W} \\
& =\left[\left|\left\langle a_{j} a_{j+\ell}\right\rangle\right|^{2}-\left|\left\langle a_{j}^{\dagger} a_{j+\ell}\right\rangle\right|^{2}\right]_{W} .
\end{aligned}
$$

Examples of $G(\ell)=G\left(j_{0}, \ell\right)$ with $j_{0}=L / 4$ are shown in Fig. 3 for a system of $L=2000$ sites and for a disorder strength $W=5$. Numerically we find that in the localized phases for model (I) when $\alpha<1, G(\ell) \sim 1 / \ell^{2}$ while for both models $G(\ell) \sim 1 / \ell^{2 \alpha}$ when $\alpha>1$. The first behaviour with a decay exponent that does not depend on $\alpha$ has been already observed in Refs! ${ }^{104 / 116}$, while the second can be explained by looking at the $\ell \rightarrow \infty$ scaling of $|C(\ell)|^{2} \sim 1 / \ell^{2 \alpha}$ in Eq. (C26) in Appendix $\mathrm{C}$

For the random interacting long-range Ising model, we compute the spin-spin correlation functions $S_{v}(\ell):=S_{v}\left(j_{0}, \ell\right)$ $(v=x, z)$ within the MBL phase with $\alpha>1$, by using a DMRG alghoritm $\frac{128}{12}$. Here we choose $j_{0}=L / 10$. For the simulations, we use up to 400 local DMRG states, 16 sweeps and we average $S_{v}(\ell)$ over 100 disorder realizations. Strikingly, we find that $S_{v}(\ell)$ decays algebraically with $\ell$ as $S_{v}(\ell) \sim \ell^{-\gamma}$ with an exponent that is consistent with $\gamma=\alpha$, in complete agreement with the discussion above for non-interacting theories. As an example, Fig. 4 (a) shows $S_{x}(\ell)$ for different values of $\alpha, W=5 \sin (\pi / 5) \approx 2.93$ and $B=0$, while Fig. 4 (b) shows
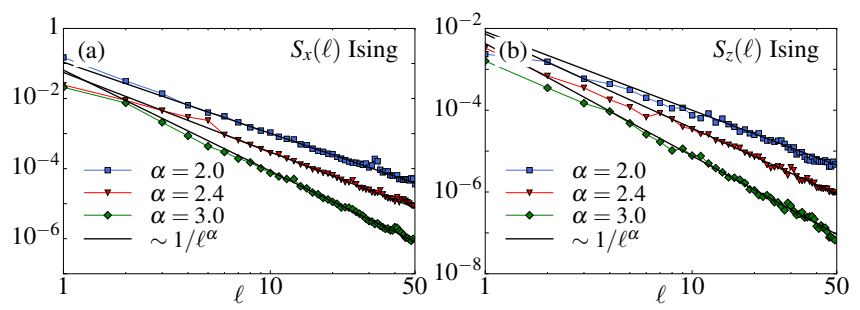

FIG. 4. (a) Correlation function $S_{x}(\ell)$ for the long-range Ising model with a random transverse field $[W=5 \sin (\pi / 5)]$ and a constant interaction term $(B=0)$ for a system of $L=100$ spins and 50 disorder realizations. (b) Correlation function $S_{z}(\ell)$ for the long-range Ising model with a random interaction $[B=5 \sin (\pi / 5)]$ and a constant magnetic field $(W=0)$. In both panels, the power-law tails are fit by the black lines scaling as $1 / \ell^{\alpha}$.

$S_{z}(\ell)$ for different values of $\alpha, W=0$ and $B=5 \sin (\pi / 5)$. The corresponding fits (continuous lines) with $1 / \ell^{\alpha}$ perfectly match the numerical results.

The demonstration of algebraic localization found in longrange couplings in the presence of disorder is a central result of this work. We argue that the fact that these results are found both for non-interacting and interacting models strongly suggests the existence of a universal behavior due to long-range coupling.

\section{LOCALIZATION OF WAVE FUNCTIONS}

Numerical results on the decay of the single-particle wave functions are obtained by considering the mean value $\Phi(\ell)=$ $\sum_{q=1}^{N}\left|g_{q, \ell-j_{M}}\right| / N$ where we average $N=L / 4$ wave functions $g_{q, \ell}$ with lowest energies, shifted by the quantity $j_{M}$ that corresponds to the lattice site where $\left|g_{q, \ell}\right|$ shows its maximum value. We average $\Phi(\ell)$ also over several disorder realizations (of the order of 500).

Figure 5 shows typical results of the decay of $\Phi(\ell)$ as a function of the distance $\ell$ within the localized phases of models (I) and (II) of Eqs. (1) [panels (a,b) and (c,d), respectively].

Remarkably, we find that the wave functions decay algebraically at long distances regardless of the strength $W$ of the disorder, mimicking the scaling of the correlation functions discussed above. However, for all $\alpha$, i.e. both $\alpha>1$ and $\alpha<1, \Phi(\ell)$ decays at large distances as $\Phi(\ell) \sim \ell^{-\gamma_{\mathrm{wf}}}$, with an exponent $\gamma_{\mathrm{wf}}$ consistent with $\gamma_{\mathrm{wf}} \sim \alpha$. This is different from the results of Ref ${ }^{[15]}$ with positional disorder, where for $\alpha<1$ one gets $\gamma_{\mathrm{wf}} \sim 2-\alpha$. For sufficiently large $\alpha>1$ this algebraic decay is preceded by an exponential decay at short distances, reminiscent of the exponentially localized states of short-range random Hamiltonians.

\section{CONCLUSIONS}

In this work we have demonstrated that couplings that decay as a power-law with distance induce an algebraic decay of correlation functions and wave functions both in non- 

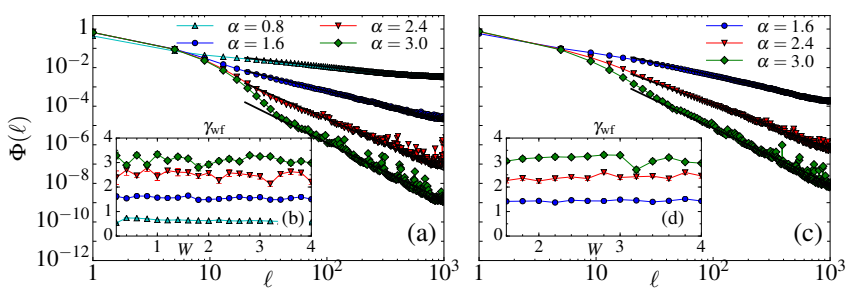

FIG. 5. (a) Decay of the averaged wave function $\Phi(\ell)$ (absolute value, see text) of localized states for the model (I): If $\alpha>1$ we find an hybrid exponential and power-law behaviour. If $\alpha<1$ the exponential part is suppressed and only the power-law tail is visible. The black lines correspond to fit of the data scaling as $1 / \ell^{\gamma_{\mathrm{wf}}}$. (b) Decay exponent $\gamma_{\mathrm{wf}}$ for the model (I) of the long-distance tail of $\Phi(\ell)$ as a function of $W$ for different values of $\alpha$. The decay exponent satisfies $\gamma_{\mathrm{wf}} \sim \alpha$ and does not show significance dependence on $W$. (c-d) Same as (a-b) but for the model (II) with random long-range pairing.

interacting and interacting models in the presence of disorder. This is in stark contrast to results expected from short-range models, and generalises recent results for the decay of wavefunctions in quadratic models. These results are of immediate interest for experiments with cold ions, molecule, Rydberg atoms and quantum emitters in cavity fields, to name a few. It is an exciting prospect to explore the properties of many-body quantum phases in the search of exotic transport phenomena with long-range interactions.

\section{ACKNOWLEDGMENTS}

P. N. thanks L. Benini for fruitful discussions. G. P. acknowledges support from ANR "ERA-NET QuantERA" Projet "RouTe" and UdS via Labex NIE. E. E. is partially supported through the project "QUANTUM" by Istituto Nazionale di Fisica Nucleare (INFN) and through the project "ALMAIDEA" by University of Bologna. The DMRG simulations were performed using the ITensor library 129 .

\section{Appendix A: Correlation in the long-range models with no disorder}

In this Appendix we summarize some results on the decay of the correlation functions for both the long-range Kitaev and Ising models in the absence of disorder.

For the long-range Kitaev model it has been shown in Refs. 104|116|125 that the one-body correlation function $C(j, \ell)=\left\langle a_{j}^{\dagger} a_{\ell}\right\rangle$ shows a different behaviour depending on the value $\alpha$ of the decay exponent of the pairing term. In the limit of a infinite system the one-body correlator takes the form of an integral on the Brillouin zone:

$$
C(j, \ell)=\left\langle a_{j}^{\dagger} a_{\ell}\right\rangle=\frac{1}{2 \pi} \int_{0}^{2 \pi} d k \frac{\mu+\cos k}{\lambda_{\alpha}(k)} e^{\mathrm{i} k(j-\ell)}
$$

where $\lambda_{\alpha}(k)=\left[(\cos k-\mu)^{2}+4 f_{\alpha}^{2}(k)\right]^{1 / 2}$ and $f_{\alpha}(k)=$
$\operatorname{ImLi}\left(e^{\mathrm{i} k}\right)$. An explicit calculation of the previous integral gives

$C(R) \equiv C(R, 0) \sim e^{-\xi R}+A(\alpha, \mu) \cdot \begin{cases}\frac{1}{R^{\alpha+1}} & \text { if } \alpha>2 ; \\ \frac{1}{R^{2 \alpha-1}} & \text { if } 1<\alpha<2 ; \\ \frac{1}{R^{2-\alpha}} & \text { if } 0<\alpha<1 .\end{cases}$

The parameter $\xi$ is the smallest real solution of $\lambda_{\alpha}(\mathrm{i} \pi+$ $\xi)=0$ and depend on $\mu$ and $\alpha$ in an implicit way and the coefficient $A(\alpha, \mu)$ can be found in Ref. ${ }^{125}$. It is possible to see that the correlation function shows a hybrid decay, i.e. exponential at short distances, followed by an algebraic tail whose decaying exponent depends on $\alpha$.

For the long-range Ising model, Ref!116 showed that the connected part of the correlation functions

$$
S_{v}(j, \ell)=\left\langle\sigma_{j}^{v} \sigma_{j+\ell}^{v}\right\rangle-\left\langle\sigma_{j}^{v}\right\rangle\left\langle\sigma_{j+\ell}^{v}\right\rangle
$$

for $v=x, z$, decay with distance with a hybrid behaviour that is exponential at short distances and algebraic at long ones.

An example is shown in Fig. 6(a) for $S_{x}(R) \equiv S_{x}(R, 0)$. The exponent $\gamma_{x}$ of the long-distance decay displays three difference behaviours: (i) for $\alpha>2$ it fulfils $\gamma_{x}=\alpha$; (ii) for $1<\alpha<2$ a hybrid decay is observed and the algebraic tail decays with an exponent $\gamma$ that depends linearly on $\alpha$ with a slope consistent with $\sim 0.55$ and (iii) for $\alpha \lesssim 1, \gamma_{x} \sim 0.25 \alpha$. The correlator $S_{z}(R)$ is shown in Fig. 6(b) and it also displays an algebraic tails that decays as $1 / R^{\gamma_{z}}$ where $\gamma_{z} \sim 2 \alpha$ for $\alpha>1$.
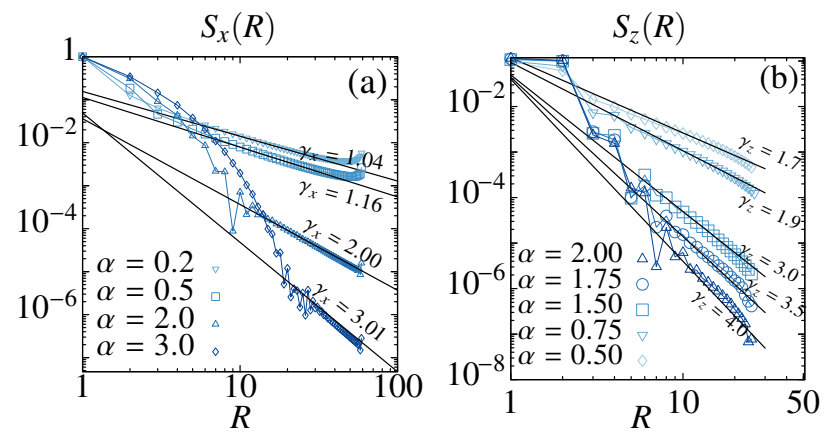

FIG. 6. (a) $S_{x}(R)$ correlation for the long-range Ising model (Eq. (5) of the main text) for $B_{i j}=W_{i j}=0(\theta=0.2 \pi$ and $L=60)$, showing the hybrid exponential and power-law behavior for $\alpha \gtrsim 1$ and a purely power-law for $\alpha \lesssim 1$. (b) $S_{z}(R)$ correlation for the longrange Ising model (Eq. (5) of the main text) for $B_{i j}=W_{j}=0$ and $\theta=0.207 \pi, L=100$ and different $\alpha$.

Appendix B: Energy scaling analysis

In this Appendix we give an analytical insight (based essentially on Ref!112) on the different behaviours of the IPR (plot- 
ted in Fig.1(a) and (b) of the main text) of the single-particle states for the Hamiltonians (I) when $\alpha>1$ and $\alpha<1$.

For the Hamiltonian (I), the random term $V_{\mathrm{I}}$, which reads $V_{\mathrm{I}}=\sum_{k k^{\prime}} J_{k, k^{\prime}}\left(e^{\mathrm{i} k}+e^{-\mathrm{i} k^{\prime}}\right) a_{k}^{\dagger} a_{k^{\prime}}$ with $J_{k, k^{\prime}}=-\sum_{j} e^{\mathrm{i}\left(k-k^{\prime}\right) j} W_{j} / L$ after a Fourier transform, couples the extended eigenmodes of $H_{0}$ to each others and it can lead to localization if the mean fluctuation $\sigma_{\mathrm{I}}^{2}:=\left\langle J_{k, k^{\prime}}^{2}\right\rangle=\delta_{k k^{\prime}} W^{2} /(3 L)$ is much larger than the level spacing $\delta \lambda_{\alpha}$ of the energies $\lambda_{\alpha}(k)$ of $H_{0}$. Here, we will restrict our attention to those states lying either at the minimum or at the maximun of $\lambda_{\alpha}(k)$. The level spacing $\delta \lambda_{\alpha}$ can be computed analytically from $\lambda_{\alpha}(k)$ and it strongly depends on $\alpha$ : (i) If $\alpha>1, \lambda_{\alpha}(k)$ is finite for all $k$ and we obtain $\lambda_{\alpha}(k) \sim k^{2}$ both at the minimum and at the maximum of the band. The corresponding level spacing thus scales with $L$ as $\delta \lambda_{\alpha>1} \sim 1 / L^{2}$ and it decays faster than $\sigma_{\mathrm{I}}$. In this case the extended states of $H_{0}$ are coupled by the random part of $V_{\mathrm{I}}$ and they will be localized. No mobility edge is then expected for $\alpha>1$. The IPR computed for this case is shown in Fig.1(a).

On the contrary, (ii) if $\alpha<1$ the energy diverges as $\lambda_{\alpha}(k) \sim$ $1 /|k|^{1-\alpha}$ and the level spacing for the high-energy states grows as $\delta \lambda_{\alpha<1} \sim L^{1-\alpha}$. The fluctuation of the random couplings $\sigma_{\mathrm{I}} \sim 1 / \sqrt{L}$ is thus suppressed by $\delta \lambda_{\alpha<1}$ and the highenergy states of $H_{0}$ remain extended. As the lowest-energy states can be localised, a single-particle mobility edge can be then expected for all $\alpha<1$. By the equality $\delta \lambda_{\alpha<1} \sim \sigma_{\text {I }}$ that defines the single particle mobility edge, it is possible to show that the number of extended states increases with $L$ as $N_{\text {ext }} \sim L^{(3 / 2-\alpha) /(2-\alpha)}$ but their fraction $N_{\text {ext }} / L$ vanishes in the thermodynamic limit 113 . The IPR for this case is shown in Fig.1(b).

\section{Appendix C: Decay of correlation functions}

In this Appendix we show how to compute the correlation function $C(j, i)=\left\langle a_{j}^{\dagger} a_{i}\right\rangle$ of the model with random hopping by perturbation theory.

\section{Correlation functions - Perturbation theory}

We recall that the Hamiltonian $H_{\mathrm{I}}$ in Eq. (1) is formed by two parts:

$$
H_{\mathrm{I}}=H_{0}+V_{\mathrm{I}}
$$

In order to compute the correlation function $C(j, i)=$ $\left\langle\Omega\left|a_{j}^{\dagger} a_{i}\right| \Omega\right\rangle$ on the ground state $|\Omega\rangle$ of $H_{\mathrm{I}}$ in Eq. (1), we first find the first-order correction $\left|\delta \Omega_{0}\right\rangle$ to the ground state $\left|\Omega_{0}\right\rangle$ of $H_{0}$ by treating $V_{\mathrm{I}}$ as a perturbation.

The first-order correction $\left|\delta \Omega_{0}\right\rangle$ to the ground state $\left|\Omega_{0}\right\rangle$ of the Hamiltonian $H_{0}$ due to the perturbation $V_{\mathrm{I}}$ is given by

$$
\left|\delta \Omega_{0}\right\rangle=\sum_{\mathbf{n}_{0}} \frac{\left\langle\mathbf{n}_{0}\left|V_{\mathrm{I}}\right| \Omega_{0}\right\rangle}{E\left(\mathbf{n}_{0}\right)-E_{0}}\left|\mathbf{n}_{0}\right\rangle
$$

where the quantities $E\left(\mathbf{n}_{0}\right)$ and $E_{0}$ are the energy of the states $\left|\mathbf{n}_{0}\right\rangle$ and of $\left|\Omega_{0}\right\rangle$, respectively and $\left|\mathbf{n}_{0}\right\rangle$ indicates an excited state of the homogeneous Hamiltonian $H_{0}$ that can be diagonalized via Fourier and Bogoliubov transformations as

$$
H_{0}=\sum_{k} \lambda_{\alpha}(k) \xi_{k}^{\dagger} \xi_{k} .
$$

The ground state $\left|\Omega_{0}\right\rangle$ of $H_{0}$ is then the vacuum of all quasiparticles $\xi_{k}$.

In Eq. C3 we have defined the single-particle energy

$$
\lambda_{\alpha}(k)=\left[(\cos k-\mu)^{2}+4 f_{\alpha}^{2}(k)\right]^{1 / 2}
$$

and the Bogolioubov quasi-particles $\xi_{k}$ that are related to the original fermionic operators $\tilde{a}_{k}$ in momentum space via

$$
\tilde{a}_{k}=v_{k} \xi_{k}-u_{k} \xi_{-k}^{\dagger}
$$

with $v_{k}=\cos \varphi(k)$ and $u_{k}=\mathrm{i} \sin \varphi(k)$ where $\tan 2 \varphi(k)=$ $f_{\alpha}(k) /[\mu-\cos k]$ and $f_{\alpha}(k)=\sum_{\ell=1}^{L-1} \sin (k \ell) / \ell^{\alpha}$. We notice that the functions $f_{\alpha}(k)$ when $L \rightarrow \infty$ become $f_{\alpha}(k)=$ $\left[\mathrm{Li}_{\alpha}\left(\mathrm{e}^{\mathrm{i} k}\right)-\mathrm{Li}_{\alpha}\left(\mathrm{e}^{-\mathrm{i} k}\right)\right] /(2 \mathrm{i})$, with $\mathrm{Li}_{\alpha}(z)=\sum_{j} z^{j} / j^{\alpha}$ a polylogarithm of order $\alpha$.

The excited states $\left|\mathbf{n}_{0}\right\rangle$ are defined by assigning a set of occupied modes $\mathbf{n}_{0}=\left\{n_{1}, n_{2}, \ldots, n_{L}\right\}$ with $n_{q}=0,1$ and then creating single quasi-particles $\xi_{q}^{\dagger}$ on the ground state $\left|\Omega_{0}\right\rangle$ if the mode $q$ is occupied

$$
\left|\mathbf{n}_{0}\right\rangle=\prod_{q=0}^{L-1}\left[\xi_{q}^{\dagger}\right]^{n_{q}}\left|\Omega_{0}\right\rangle
$$

The first-order correction $\left|\delta \Omega_{0}\right\rangle$ can now be obtained from Eq. (C2) and the true ground state $|\Omega\rangle$ becomes

$$
\begin{aligned}
|\Omega\rangle & =|\widetilde{\Omega}\rangle+\mathscr{O}\left(W^{2}\right) \\
& =\left|\Omega_{0}\right\rangle+\left|\delta \Omega_{0}\right\rangle+\mathscr{O}\left(W^{2}\right) \\
& =\left|\Omega_{0}\right\rangle-\sum_{k k^{\prime}} J_{k, k^{\prime}} A\left(k, k^{\prime}\right) \xi_{k}^{\dagger} \xi_{k^{\prime}}^{\dagger}\left|\Omega_{0}\right\rangle+\mathscr{O}\left(W^{2}\right),
\end{aligned}
$$

where we have defined $J_{k, k^{\prime}}=-\sum_{j} e^{\mathrm{i}\left(k-k^{\prime}\right) j} W_{j} / L$ and $A\left(k, k^{\prime}\right)=2\left(\mathrm{e}^{\mathrm{i} k}+\mathrm{e}^{-\mathrm{i} k^{\prime}}\right) v_{k} u_{k^{\prime}}^{*} /\left[\lambda(k)+\lambda\left(k^{\prime}\right)\right]$.

On a single disorder realization the correlation function $\left\langle\Omega\left|a_{j}^{\dagger} a_{i}\right| \Omega\right\rangle$ takes the form

$$
\begin{aligned}
\left\langle\Omega\left|a_{j}^{\dagger} a_{i}\right| \Omega\right\rangle & =\left\langle\Omega_{0}\left|a_{j}^{\dagger} a_{i}\right| \Omega_{0}\right\rangle+\left\langle\delta \Omega_{0}\left|a_{j}^{\dagger} a_{i}\right| \Omega_{0}\right\rangle \\
& +\left\langle\Omega_{0}\left|a_{j}^{\dagger} a_{i}\right| \delta \Omega_{0}\right\rangle+\left\langle\delta \Omega_{0}\left|a_{j}^{\dagger} a_{i}\right| \delta \Omega_{0}\right\rangle .
\end{aligned}
$$

If we now average Eq. (C8) over many disorder realizations, the cross terms $\left\langle\delta \Omega_{0}\left|a_{j}^{\dagger} a_{i}\right| \Omega_{0}\right\rangle$ and $\left\langle\Omega_{0}\left|a_{j}^{\dagger} a_{i}\right| \delta \Omega_{0}\right\rangle$ vanish as, due to the correction $\left|\delta \Omega_{0}\right\rangle$, only one random term $W_{j}$ (that has mean value zero) appears in them. Therefore we get

$$
\left\langle\Omega\left|a_{j}^{\dagger} a_{i}\right| \Omega\right\rangle_{W}=\left\langle\Omega_{0}\left|a_{j}^{\dagger} a_{i}\right| \Omega_{0}\right\rangle+\left\langle\delta \Omega_{0}\left|a_{j}^{\dagger} a_{i}\right| \delta \Omega_{0}\right\rangle_{W} .
$$

The first term of the r.h.s. of Eq. C9] corresponds to the correlator for a homogenous translationally-invariant system. By rewriting $a_{j}^{\dagger}$ and $a_{i}$ in momentum space and by using Eq. (C5) recalling that $\xi_{k}\left|\Omega_{0}\right\rangle=0$ we obtain

$$
C_{0}(\ell):=\left\langle\Omega_{0}\left|a_{j}^{\dagger} a_{i}\right| \Omega_{0}\right\rangle=\frac{1}{L} \sum_{k} \mathrm{e}^{\mathrm{i} k \ell} R_{0}(k)
$$


where $\ell=j-i$ and $R_{0}(k)=\left|u_{k}\right|^{2}$.

In the second term of the r.h.s. of Eq. (C9), as we are averaging on the disorder configurations, we can expect that the disorder average $\left\langle\delta \Omega_{0}\left|a_{j}^{\dagger} a_{i}\right| \delta \Omega_{0}\right\rangle_{W}$ will be translationally invariant, i.e. it will depend on the relative distance $\ell=j-i$ while the terms that depend on $i$ and $j$ separately will average out to zero (see $\S 6.5$ in Ref. ${ }^{131}$ or $\S 12.3$ in Ref. ${ }^{132}$ ). By keeping only the terms that depend on $\ell$, after rewriting $a_{j}^{\dagger}$ and $a_{i}$ in momentum space and using again Eq. C5 recalling that $\xi_{k}\left|\Omega_{0}\right\rangle=0$, the second term becomes

$$
C_{1}(\ell):=\left\langle\delta \Omega_{0}\left|a_{j}^{\dagger} a_{i}\right| \delta \Omega_{0}\right\rangle_{W}=\frac{W^{2}}{3 L} \sum_{k} \mathrm{e}^{\mathrm{i} k \ell} R_{1}(k)
$$

where

$$
\begin{aligned}
R_{1}(k) & =c\left|u_{k}\right|^{2}+U(k)\left|u_{k}\right|^{2}-V(k)\left|v_{k}\right|^{2}, \\
c & =\sum_{p} A(p, p)^{2}-\sum_{p_{1} p_{2}} A\left(p_{1}, p_{2}\right) A\left(p_{2}, p_{1}\right), \\
U(k) & =2 \sum_{p} A(p,-k) A(-k, p) \\
& =-\frac{f_{\alpha}(k)}{\lambda_{\alpha}(k)} \sum_{p} \frac{2+2 \cos (p-k)}{\left(\lambda_{\alpha}(k)+\lambda_{\alpha}(p)\right)^{2}} \frac{f_{\alpha}(p)}{\lambda_{\alpha}(p)}, \\
V(k) & =U(-k)
\end{aligned}
$$

We note that the quantity $c$ does not depend on $k$.

\section{Correlation functions - Asymptotic behavior}

In this Section we show how the two correlators $C_{0}(\ell)$ and $C_{1}(\ell)$ behave asymptotically for $\ell \rightarrow \infty$.

Let us consider $C_{0}(\ell)$ in Eq. C10] first. In the limit $L \rightarrow \infty$ we can replace the summation with an integral

$$
C_{0}(\ell)=\frac{1}{2 \pi} \int_{-\infty}^{\infty} \mathrm{d} k \mathrm{e}^{\mathrm{i} k \ell} R_{0}(k) .
$$

The asymptotic behavior of $C_{0}(\ell)$ for $\ell \rightarrow \infty$ can be computed by considering the integrals $I_{0}^{+}$and $I_{0}^{-}$on the complex plane in Fig. 7 that are

$$
I_{0}^{ \pm}=\frac{1}{2 \pi} \int_{s_{ \pm}} \mathrm{d} z \mathrm{e}^{\mathrm{i} z \ell} R_{0}(z)+\frac{1}{2 \pi} \int_{\Gamma_{ \pm}} \mathrm{d} z \mathrm{e}^{\mathrm{i} z \ell} R_{0}(z)+\frac{1}{2 \pi} \int_{0}^{\infty} \mathrm{d} k \mathrm{e}^{\mathrm{i} k \ell} R_{0}(k)
$$

where we have chosen to put the branch cut of the complex logarithm [see the expansion of the polylogarithm in Eq. [C19]] on the imaginary positive axis.

By sending the radius $r$ of the circles $\Gamma_{ \pm}$to infinity and by neglecting possible residues inside the integration contour that will contribute only with exponential decaying terms we have

$$
\begin{aligned}
C_{0}(\ell) & =-\frac{1}{2 \pi} \int_{s_{+}} \mathrm{d} z \mathrm{e}^{\mathrm{i} z \ell} R_{0}(z)-\frac{1}{2 \pi} \int_{s_{-}} \mathrm{d} z \mathrm{e}^{\mathrm{i} z \ell} R_{0}(z) \\
& =\frac{\mathrm{i}}{2 \pi} \int_{0}^{\infty} \mathrm{d} y \mathrm{e}^{-y \ell} R_{0}(\varepsilon+\mathrm{i} y)-\frac{\mathrm{i}}{2 \pi} \int_{-\infty}^{0} \mathrm{~d} y \mathrm{e}^{-y \ell} R_{0}(-\varepsilon+\mathrm{i} y) \\
& =\frac{1}{\pi} \int_{0}^{\infty} \mathrm{d} y \mathrm{e}^{-y \ell} \operatorname{Im} R_{0}(\mathrm{i} y)
\end{aligned}
$$

where on the lines $s_{ \pm}$the complex variable is $z= \pm \varepsilon+\mathrm{i} y$ with $\varepsilon$ a small positive parameter that we send to zero.
We are able now to evaluate the asymptotic behavior of $C_{0}(\ell)$ by computing the $y \rightarrow 0$ part of $\operatorname{Im}\left[R_{0}(\mathrm{i} y)\right]$ and then integrating the last equality in Eq. (C18). This is done by recalling that the polylogarithm admits the series expansion ${ }^{133 \mid 134}$ for a general complex number $z$ as

$$
\operatorname{Li}_{\alpha}(z)=\Gamma(1-\alpha)\left(\ln \frac{1}{z}\right)^{\alpha-1}+\sum_{n=0}^{\infty} \zeta(\alpha-n) \frac{(\ln z)^{n}}{n !}
$$

that makes them non-analytical due to the presence of the complex logarithm and the power-law. In Eq. (C19), $\Gamma(x)$ and $\zeta(x)$ are the Euler gamma function and the Riemann zeta function, respectively.

By using the series expansion of the polylogarithms from Eq. (C19) we can obtain the function $R_{0}$ (iy) on the imaginary axis:

$$
R_{0}(\mathrm{i} y)=\frac{\mu-\cosh y}{2 \lambda_{\alpha}(\mathrm{i} y)} \sim \frac{\mu-1}{2 \sqrt{(\mu-1)^{2}-\Gamma^{2}(1-\alpha)\left(\mathrm{e}^{\mathrm{i} \pi \alpha}+1\right)^{2} y^{2 \alpha-2}-4 \Gamma(1-\alpha)\left(\mathrm{e}^{\mathrm{i} \pi \alpha}+1\right) \zeta(\alpha-1) y^{\alpha}}}
$$


The previous equation in the limit $y \rightarrow 0$ gives

$$
\operatorname{Im} R_{0}(\mathrm{i} y)= \begin{cases}y^{1-\alpha} & \text { for } \alpha<1 \\ y^{2 \alpha-2} & \text { for } 1<\alpha<2 \\ y^{\alpha} & \text { for } \alpha>2\end{cases}
$$

and, after performing the last integral in Eq. [C18, the asymptotic behavior of $C_{0}$ turns out to be

$$
C_{0}(\ell) \sim \begin{cases}1 / \ell^{2-\alpha} & \text { for } \alpha<1 \\ 1 / \ell^{2 \alpha-1} & \text { for } 1<\alpha<2 \\ 1 / \ell^{\alpha+1} & \text { for } \alpha>2\end{cases}
$$

For the correlator $C_{1}(\ell)$ in Eq. C11 we can use the same contour in Fig. 7 and get

$$
C_{1}(\ell)=\frac{W^{2}}{3 \pi} \int_{0}^{\infty} \mathrm{d} y \mathrm{e}^{-y \ell} \operatorname{Im} R_{1}(\mathrm{i} y) .
$$

For the asymptotic behaviour of $C_{1}(\ell)$, we need again the $y \rightarrow 0$ part of $R_{1}(\mathrm{i} y)$. Let us start by noting that from Eqs. (C14) and (C15) the $y \rightarrow 0$ part of both $U(\mathrm{iy})$ and $V(\mathrm{i} y)$ is given by

$$
\begin{aligned}
\operatorname{Im}\left[U(\mathrm{i} y)\left|u_{\mathrm{iy} y}\right|^{2}\right] & \sim \operatorname{Im}\left[V(\mathrm{i} y)\left|v_{\mathrm{iy}}\right|^{2}\right] \\
& \sim \operatorname{Im} \frac{f_{\alpha}(\mathrm{i} y)(\mu-\cosh y)}{\lambda_{\alpha}^{2}(\mathrm{i} y)} \\
& \sim \begin{cases}y^{1-\alpha} & \text { for } \alpha<1 \\
y^{\alpha-1} & \text { for } \alpha>1 .\end{cases}
\end{aligned}
$$

The previous equation, by considering also the contribution coming from $c\left|u_{\mathrm{iy}}\right|^{2}$ [see Eq. (C12)], gives

$$
\operatorname{Im} R_{1}(\mathrm{i} y) \sim \begin{cases}y^{1-\alpha} & \text { for } \alpha<1 \\ y^{\alpha-1} & \text { for } \alpha>1\end{cases}
$$

and after integrating Eq. (C23), we finally get the correlator

$$
C_{1}(\ell)= \begin{cases}W^{2} / \ell^{2-\alpha} & \text { for } \alpha<1 \\ W^{2} / \ell^{\alpha} & \text { for } \alpha>1\end{cases}
$$

The asymptotic behavior coming from Eqs. (C22), can be checked by computing the correlator $C(\ell)$ numerically as reported in Fig. 2(a) of the main text. Remarkably, the values of the decay exponents of the power-law tails do not depend on the disorder strength $W$ as shown in Fig. 8 where we plot the decay exponents of $C(\ell)$ as a function of $W$ for different values of $\alpha$. For completeness we show also the decay exponent of the correlation function $C(\ell)$ for the model (II) with random long-range pairing.

\section{Validity of the perturbation theory}

In this Section we give some details on the validity of the perturbation theory in approximating the true ground state of

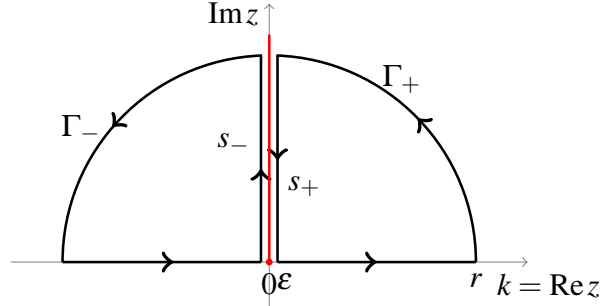

FIG. 7. Integration contour for evaluating the asymptotic behaviors of the correlators $C_{0}(\ell)$ in Eq. $(\mathrm{C} 18)$ and $C_{1}(\ell)$ in Eq. (C23).
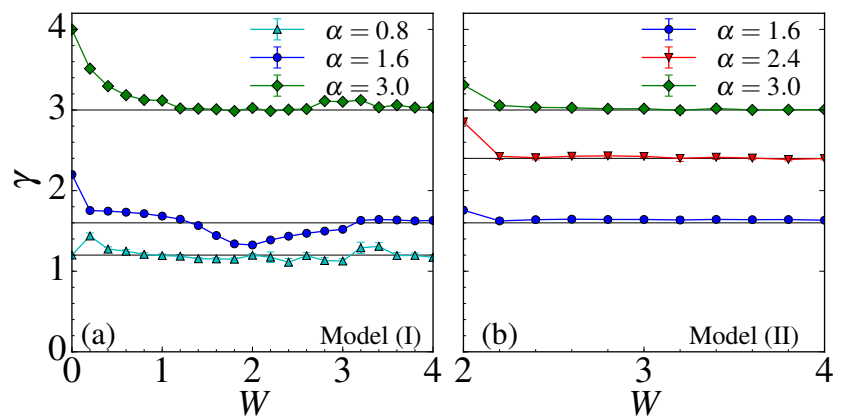

FIG. 8. (a) Decay exponent $\gamma$ of the long-distance tail of the correlation function $C(\ell)$ for the model (I) as a function of $W$ and for $\alpha=0.8$ (cyan triangles), $\alpha=1.6$ (blue circles), $\alpha=3.0$ (green diamonds). If $W>0$, the decay exponent satisfies $\gamma \sim \alpha$ for $\alpha>1$ and $\gamma \sim 2-\alpha$ for $\alpha<1$ and it does not show significance dependence on $W$. These data are obtained by computing the correlation function $C(\ell)$ numerically from the full random Hamiltonian in Eq. (1) and then by fitting the long-range decaying tail of $C(\ell)$ with $1 / \ell^{\gamma}$. The black lines represent the expected exponents: $\gamma=1.2$ for $\alpha=0.8$, $\gamma=1.6$ for $\alpha=1.6, \gamma=3.0$ for $\alpha=3.0$. (b) Same as panel (a) but for the localized phase (for $W \gtrsim 2$ ) of model (II).

$H_{\mathrm{I}}$ by the state $|\widetilde{\Omega}\rangle$ in Eq. [C7]. To this end, we will compare the energy $E_{|\Omega\rangle}$ of the ground state of the Hamiltonian $H_{\text {I }}$ computed numerically with the energy $E_{|\widetilde{\Omega}\rangle}$ of the state $|\widetilde{\Omega}\rangle=\left|\Omega_{0}\right\rangle+\left|\delta \Omega_{0}\right\rangle$ coming from the first order correction given in Eq. (C7).

The energy $E_{|\widetilde{\Omega}\rangle}$ can be obtained by considering that the first order correction to a wave function gives a second order correction to the energy 135 . Therefore, the energy for the perturbed state $|\widetilde{\Omega}\rangle$ is given by

$$
E_{|\widetilde{\Omega}\rangle}=E_{\left|\Omega_{0}\right\rangle}+\sum_{\mathbf{n}_{0}} \frac{\left|\left\langle\Omega_{0}\left|V_{\mathrm{I}}\right| \mathbf{n}_{0}\right\rangle\right|^{2}}{E_{\left|\Omega_{0}\right\rangle}-E_{\left|\mathbf{n}_{0}\right\rangle}}
$$

where $E_{\left|\Omega_{0}\right\rangle}$ is the ground state energy of the Hamiltonian $H_{0}$, $\left|\mathbf{n}_{0}\right\rangle$ denotes the excited states of $H_{0}$ from Eq. (C6) and $E_{\left|\mathbf{n}_{0}\right\rangle}$ denotes their energy.

By computing the scalar product $\left\langle\mathbf{n}_{0}\left|V_{\mathrm{I}}\right| \Omega_{0}\right\rangle$ we get

$$
E_{|\widetilde{\Omega}\rangle}=E_{\left|\Omega_{0}\right\rangle}-\sum_{q_{1}, q_{2}} \frac{\left|\mathscr{E}_{q_{1}, q_{2}}\right|^{2}}{\lambda_{\alpha}\left(q_{1}\right)+\lambda_{\alpha}\left(q_{2}\right)}
$$

where $\mathscr{E}_{q_{1}, q_{2}}=2 J_{q_{1}, q_{2}} \mathrm{e}^{\mathrm{i} q_{1}} v_{q_{1}} u_{q_{2}}^{*}+$ h.c. where $v_{q_{1}}$ and $u_{q_{2}}$ are 

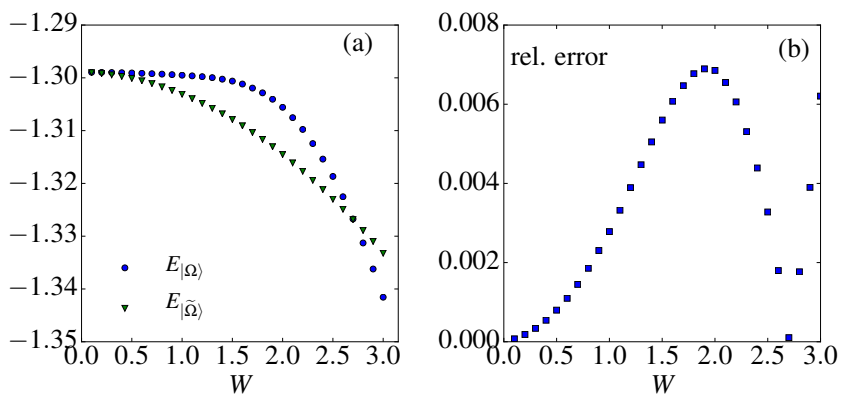

FIG. 9. (a) Energy density $E_{|\Omega\rangle} / L$ of the ground state of the Hamiltonian $H_{\mathrm{I}}$ computed numerically (blue circles) and the energy $E_{\mid \widetilde{\left.\Omega_{0}\right\rangle}} / L$ (green triangles) computed by perturbation theory. (b) Relative error defined as $\left|1-E_{|\widetilde{\Omega}\rangle} / E_{|\Omega\rangle}\right|$. It is possible to see that the difference between the true ground state energy and the perturbed one is bounded by $8 \cdot 10^{-3}$ when the disorder strength satisfies $0 \leq W \leq 3$. For both panels we consider $\alpha=3.0$ and a system of $L=400$ sites and after averaging 200 disorder realizations.
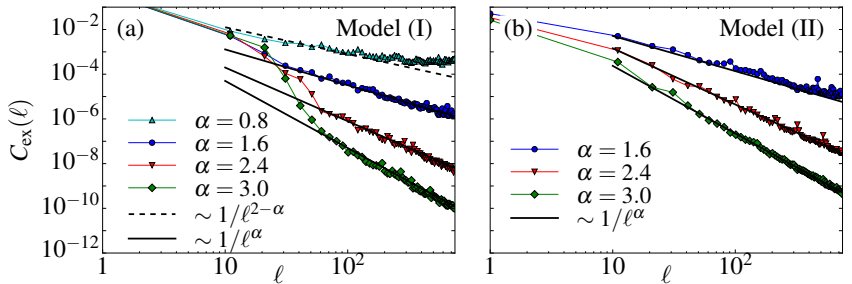

FIG. 10. Correlation function $C_{\mathrm{ex}}(\ell)$ computed on the an excited state at the middle of the energy band for the model (I) as a function of the lattice site $\ell$ for different values of $\alpha$ and for $W=5, L=1000$ and 200 disorder realizations. The continuous lines are guides to the eye and show that the power-law tails scales as $1 / \ell^{2-\alpha}$ (dashed) and $1 / \ell^{2 \alpha}$ (solid) also for that excite state. (b) Same as panel (a) but for the model (II). defined after Eq. C5 and $J_{q_{1}, q_{2}}$ after Eq. (C7). The left panel of Fig. 9 shows the values of the energy density $E_{|\Omega\rangle} / L$ of the ground state of the Hamiltonian $H_{\mathrm{I}}$ computed numerically (blue circles) with the energy $E_{\left|\widetilde{\Omega_{0}}\right\rangle} / L$ (green triangles), while the right panel shows the relative error defined as $\left|1-E_{|\widetilde{\Omega}\rangle} / E_{|\Omega\rangle}\right|$ for a system of $L=400$ sites and after averaging 200 disorder realizations for $\alpha=3.0$. It is possible to see that the difference between the true ground state energy and the perturbed one is bounded by $8 \cdot 10^{-3}$ when the disorder strength satisfies $0 \leq W \leq 3$.

\section{Correlators on excited states}

In order to show that the algebraic tail of the correlators $a_{j}^{\dagger} a_{\ell}$ is not peculiar only to the ground state, but it is typical also for the excited states, we computed the correlation $C_{\mathrm{ex}}(j, \ell)=\left\langle\Omega\left|\eta_{q} a_{j}^{\dagger} a_{\ell} \eta_{q}^{\dagger}\right| \Omega\right\rangle$ for the excited state $\eta_{q}^{\dagger}|\Omega\rangle$. In Fig. 10 we plot $C_{\mathrm{ex}}(j, \ell)$ for an excited state that lies in the middle of the energy band for both the models (I) and (II) and for different values of $\alpha$. It is possible to see that the algebraic tail is present also in this case.
* davide.vodola@gmail.com

$\dagger$ pupillo@unistra.fr

1 P. W. Anderson, Phys. Rev. 109, 1492 (1958)

2 G. Roati, C. D'Errico, L. Fallani, M. Fattori, C. Fort, M. Zaccanti, G. Modugno, M. Modugno, and M. Inguscio, Nature (London) 453, 895 (2008)

3 J. Billy, V. Josse, Z. Zuo, A. Bernard, B. Hambrecht, P. Lugan, D. Clement, L. Sanchez-Palencia, P. Bouyer, and A. Aspect, Nature (London) 453, 891 (2008).

4 S. S. Kondov, W. R. McGehee, J. J. Zirbel, and B. DeMarco, Science 334, 66 (2011)

5 F. Jendrzejewski, A. Bernard, K. Muller, P. Cheinet, V. Josse, M. Piraud, L. Pezze, L. Sanchez-Palencia, A. Aspect, and P. Bouyer, Nat. Phys. 8, 398 (2012)

${ }^{6}$ M. Schreiber, S. S. Hodgman, P. Bordia, H. P. Lüschen, M. H. Fischer, R. Vosk, E. Altman, U. Schneider, and I. Bloch, Science 349, 842 (2015).

J. Smith, A. Lee, P. Richerme, B. Neyenhuis, P. W. Hess, P. Hauke, M. Heyl, D. A. Huse, and C. Monroe, Nat. Phys. 12, 907 (2016)
${ }^{8}$ P. Bordia, H. P. Lüschen, S. S. Hodgman, M. Schreiber, I. Bloch, and U. Schneider, Phys. Rev. Lett. 116, 140401 (2016)

9 P. A. Lee and T. V. Ramakrishnan, Rev. Mod. Phys. 57, 287 (1985)

${ }^{10}$ B. L. Altshuler, Y. Gefen, A. Kamenev, and L. S. Levitov, Phys. Rev. Lett. 78, 2803 (1997).

11 D. Basko, I. Aleiner, and B. Altshuler, Ann. Phys. 321, 1126 (2006)

12 V. Oganesyan and D. A. Huse, Phys. Rev. B 75, 155111 (2007)

13 I. V. Gornyi, A. D. Mirlin, and D. G. Polyakov, Phys. Rev. Lett. 95, 206603 (2005)

${ }^{14}$ M. Žnidarič, T. c. v. Prosen, and P. Prelovšek, Phys. Rev. B 77, $064426(2008)$

15 J. Biddle, B. Wang, D. J. Priour, and S. Das Sarma, Phys. Rev. A 80, 021603 (2009)

10 J. Biddle and S. Das Sarma, Phys. Rev. Lett. 104, 070601 (2010)

17 J. Biddle, D. J. Priour, B. Wang, and S. Das Sarma, Phys. Rev. B 83, 075105 (2011).

18 A. Pal and D. A. Huse, Phys. Rev. B 82, 174411 (2010)

19 A. D. Luca and A. Scardicchio, EPL (Europhys. Lett.) 101, 37003 (2013). 
20 R. Vosk and E. Altman, Phys. Rev. Lett. 110, 067204 (2013)

21 Y. Bar Lev and D. R. Reichman, Phys. Rev. B 89, 220201 (2014)

22 Y. Bar Lev, G. Cohen, and D. R. Reichman, Phys. Rev. Lett. 114, 100601 (2015)

23 D. J. Luitz, N. Laflorencie, and F. Alet, Phys. Rev. B 91, 081103 (2015)

${ }^{24}$ D. J. Luitz, Phys. Rev. B 93, 134201 (2016)

25 P. Naldesi, E. Ercolessi, and T. Roscilde, SciPost Phys. 1, 010 (2016)

26 R. Nandkishore, S. Gopalakrishnan, and D. A. Huse, Phys. Rev. B 90, 064203 (2014)

21 I. V. Gornyi, A. D. Mirlin, M. Müller, and D. G. Polyakov, Annalen der Physik 529, 1600365 (2017)

28 A. C. Potter and R. Vasseur, Phys. Rev. B 94, 224206 (2016)

29 J. T. Cantin, T. Xu, and R. V. Krems, Phys. Rev. B 98, 014204 (2018)

30 D. J. Luitz and Y. Bar Lev, Phys. Rev. A 99, 010105 (2019)

31 X.-L. Deng, D. Porras, and J. I. Cirac, Phys. Rev. A 72, 063407 (2005)

32 M. Kastner, Phys. Rev. Lett. 104, 240403 (2010)

33 Z.-X. Gong, M. Foss-Feig, S. Michalakis, and A. V. Gorshkov, Phys. Rev. Lett. 113, 030602 (2014)

34 M. Foss-Feig, Z.-X. Gong, C. W. Clark, and A. V. Gorshkov, Phys. Rev. Lett. 114, 157201 (2015)

35 P. Hauke and L. Tagliacozzo, Phys. Rev. Lett. 111, 207202 (2013)

36 J. Eisert, M. van den Worm, S. R. Manmana, and M. Kastner, Phys. Rev. Lett. 111, 260401 (2013)

37 J. Schachenmayer, B. P. Lanyon, C. F. Roos, and A. J. Daley, Phys. Rev. X 3, 031015 (2013)

38 D. Métivier, R. Bachelard, and M. Kastner, Phys. Rev. Lett. 112, 210601 (2014)

39 M. Kastner and M. van den Worm, Physica Scripta 2015, 014039 (2015)

40 L. Cevolani, G. Carleo, and L. Sanchez-Palencia, Phys. Rev. A 92, 041603 (2015).

41 L. Cevolani, G. Carleo, and L. Sanchez-Palencia, New Journal of Physics 18, 093002 (2016)

42 R. J. Bettles, J. Minář, C. S. Adams, I. Lesanovsky, and B. Olmos, Phys. Rev. A 96, 041603 (2017)

43 S. Hernández-Santana, C. Gogolin, J. I. Cirac, and A. Acín, Phys. Rev. Lett. 119, 110601 (2017)

44 I. Frérot, P. Naldesi, and T. Roscilde, Phys. Rev. B 95, 245111 (2017)

45 I. Frérot, P. Naldesi, and T. Roscilde, Phys. Rev. Lett. 120, 050401 (2018)

40 C. Schneider, D. Porras, and T. Schätz, Reports on Progress in Physics 75, 024401 (2012)

$4 /$ P. Richerme, Z.-X. Gong, A. Lee, C. Senko, J. Smith, M. FossFeig, S. Michalakis, A. V. Gorshkov, and C. Monroe, Nature (London) 511, 198 (2014)

${ }^{4 \gamma}$ P. Jurcevic, B. P. Lanyon, P. Hauke, C. Hempel, P. Zoller, R. Blatt, and C. F. Roos, Nature (London) 511, 202 (2014)

49 J. W. Britton, B. C. Sawyer, A. C. Keith, C. C. J. Wang, J. K. Freericks, H. Uys, M. J. Biercuk, and J. J. Bollinger, Nature (London) 484, 489 (2012)

50 A. Bermudez, T. Schäetz, and M. B. Plenio, Phys. Rev. Lett. 110, $110502(2013)$

${ }^{5}$ E. Shahmoon and G. Kurizki, Phys. Rev. A 87, 033831 (2013)

52 J. S. Douglas, H. Habibian, C. L. Hung, A. V. Gorshkov, H. J. Kimble, and D. E. Chang, Nat. Photonics 9, 326 (2015).

53 M. Litinskaya, E. Tignone, and G. Pupillo, Scientific Reports 6, 25630 (2016)
54 V. D. Vaidya, Y. Guo, R. M. Kroeze, K. E. Ballantine, A. J. Kollár, J. Keeling, and B. L. Lev, Phys. Rev. X 8, 011002 (2018)

55 H. Kadau, M. Schmitt, M. Wenzel, C. Wink, T. Maier, I. FerrierBarbut, and T. Pfau, Nature 530, 194 EP (2016)

56 S. Lepoutre, L. Gabardos, K. Kechadi, P. Pedri, O. Gorceix, E. Maréchal, L. Vernac, and B. Laburthe-Tolra, Phys. Rev. Lett. 121, $013201(2018)$

${ }^{\prime}$ S. Baier, D. Petter, J. H. Becher, A. Patscheider, G. Natale, L. Chomaz, M. J. Mark, and F. Ferlaino, Phys. Rev. Lett. 121, $093602(2018)$

58 Y. Tang, W. Kao, K.-Y. Li, and B. L. Lev, Phys. Rev. Lett. 120, $230401(2018)$

${ }^{59}$ H. Weimer, R. Löw, T. Pfau, and H. P. Büchler, Phys. Rev. Lett. 101, 250601 (2008)

${ }^{60}$ M. Saffman, T. G. Walker, and K. Mølmer, Rev. Mod. Phys. 82, 2313 (2010)

${ }^{61}$ M. Viteau, P. Huillery, M. G. Bason, N. Malossi, D. Ciampini, O. Morsch, E. Arimondo, D. Comparat, and P. Pillet, Phys. Rev. Lett. 109, 053002 (2012)

${ }^{62}$ P. Schauß, M. Cheneau, M. Endres, T. Fukuhara, S. Hild, A. Omran, T. Pohl, C. Gross, S. Kuhr, and I. Bloch, Nature (London) 491, 87 (2012)

${ }^{63}$ C. Carr, R. Ritter, C. G. Wade, C. S. Adams, and K. J. Weatherill, Phys. Rev. Lett. 111, 113901 (2013)

64 D. Barredo, S. Ravets, H. Labuhn, L. Béguin, A. Vernier, F. Nogrette, T. Lahaye, and A. Browaeys, Phys. Rev. Lett. 112, $183002(2014)$

65 J. B. Balewski, A. T. Krupp, A. Gaj, S. Hofferberth, R. Löw, and T. Pfau, New Journal of Physics 16, 063012 (2014)

66 Y. Y. Jau, A. M. Hankin, T. Keating, I. H. Deutsch, and G. W. Biedermann, Nature Physics 12, 71 (2015).

67 T. M. Weber, M. Höning, T. Niederprüm, T. Manthey, O. Thomas, V. Guarrera, M. Fleischhauer, G. Barontini, and H. Ott, Nature Physics 11, 157 EP (2015)

68 R. Faoro, C. Simonelli, M. Archimi, G. Masella, M. M. Valado, E. Arimondo, R. Mannella, D. Ciampini, and O. Morsch, Phys. Rev. A 93, 030701 (2016)

${ }^{69}$ H. Labuhn, D. Barredo, S. Ravets, S. de Léséleuc, T. Macrì, T. Lahaye, and A. Browaeys, Nature 534, 667 (2016)

70 H. Gorniaczyk, C. Tresp, P. Bienias, A. Paris-Mandoki, W. Li, I. Mirgorodskiy, H. P. Büchler, I. Lesanovsky, and S. Hofferberth, Nature Communications 7, 12480 (2016)

71 J. Zeiher, R. van Bijnen, P. Schauß, S. Hild, J.-y. Choi, T. Pohl, I. Bloch, and C. Gross, Nature Physics 12, 1095 (2016)

72 H. Bernien, S. Schwartz, A. Keesling, H. Levine, A. Omran, H. Pichler, S. Choi, A. S. Zibrov, M. Endres, M. Greiner, V. Vuletić, and M. D. Lukin, Nature 551, 579 (2017)

73 A. Piñeiro Orioli, A. Signoles, H. Wildhagen, G. Günter, J. Berges, S. Whitlock, and M. Weidemüller, Phys. Rev. Lett. 120, 063601 (2018)

${ }^{14}$ B. Yan, S. A. Moses, B. Gadway, J. P. Covey, K. R. A. Hazzard, A. M. Rey, D. S. Jin, and J. Ye, Nature (London) 501, 521 (2013)

15 K. R. A. Hazzard, M. van den Worm, M. Foss-Feig, S. R. Manmana, E. G. Dalla Torre, T. Pfau, M. Kastner, and A. M. Rey, Phys. Rev. A 90, 063622 (2014)

76 L. Reichsöllner, A. Schindewolf, T. Takekoshi, R. Grimm, and H.-C. Nägerl, Phys. Rev. Lett. 118, 073201 (2017)

77 G. A. Álvarez, D. Suter, and R. Kaiser, Science 349, 846 (2015)

78 W. R. Anderson, J. R. Veale, and T. F. Gallagher, Phys. Rev. Lett. 80, 249 (1998)

${ }^{19}$ W. R. Anderson, M. P. Robinson, J. D. Martin, and T. F. Gallagher, Phys. Rev. A 65, 063404 (2002)

80 G. D. Scholes and G. Rumbles, Nature Materials 5, 683 (2006) 
81 F. Dubin, R. Melet, T. Barisien, R. Grousson, L. Legrand, M. Schott, and V. Voliotis, Nature Physics 2, 32 (2005)

82 F. Dubin, J. Berrehar, R. Grousson, M. Schott, and V. Voliotis, Phys. Rev. B 73, 121302 (2006)

83 X. P. Vögele, D. Schuh, W. Wegscheider, J. P. Kotthaus, and A. W. Holleitner, Phys. Rev. Lett. 103, 126402 (2009)

84 S. Wüster, C. Ates, A. Eisfeld, and J. M. Rost, New Journal of Physics 13, 073044 (2011)

${ }^{\text {} 5 ~ G . ~ G u ̈ n t e r, ~ H . ~ S c h e m p p, ~ M . ~ R o b e r t-d e ~ S a i n t-V i n c e n t, ~ V . ~ G a v r y u-~}$ sev, S. Helmrich, C. S. Hofmann, S. Whitlock, and M. Weidemüller, Science 342, 954 (2013)

86 F. Robicheaux and N. M. Gill, Phys. Rev. A 89, 053429 (2014)

87 D. W. Schönleber, A. Eisfeld, M. Genkin, S. Whitlock, and S. Wüster, Phys. Rev. Lett. 114, 123005 (2015)

${ }^{88}$ H. Schempp, G. Günter, S. Wüster, M. Weidemüller, and S. Whitlock, Phys. Rev. Lett. 115, 093002 (2015)

89 D. Barredo, H. Labuhn, S. Ravets, T. Lahaye, A. Browaeys, and C. S. Adams, Phys. Rev. Lett. 114, 113002 (2015)

90 I. Rosenberg, D. Liran, Y. Mazuz-Harpaz, K. West, L. Pfeiffer, and R. Rapaport, Science Advances 4 (2018), 10.1126/sciadv.aat 8880

91 F. Pientka, L. I. Glazman, and F. von Oppen, Phys. Rev. B 88, $155420(2013)$

${ }^{92}$ F. Pientka, L. I. Glazman, and F. von Oppen, Phys. Rev. B 89, 180505 (2014)

93 D. T. Liu, J. Shabani, and A. Mitra, Phys. Rev. B 97, 235114 (2018)

${ }^{94}$ D. T. Liu, J. Shabani, and A. Mitra, Phys. Rev. B 99, 094303 (2019)

95 A. Y. Kitaev, Physics-Uspekhi 44, 131 (2001)

96 F. Liu, R. Lundgren, P. Titum, G. Pagano, J. Zhang, C. Monroe, and A. V. Gorshkov, Phys. Rev. Lett. 122, 150601 (2019)

97 J. Zhang, P. W. Hess, A. Kyprianidis, P. Becker, A. Lee, J. Smith, G. Pagano, I. D. Potirniche, A. C. Potter, A. Vishwanath, N. Y. Yao, and C. Monroe, Nature 543, 217 EP (2017)

98 O. Viyuela, D. Vodola, G. Pupillo, and M. A. Martin-Delgado, Phys. Rev. B 94, 125121 (2016)

99 Z.-X. Gong, M. F. Maghrebi, A. Hu, M. L. Wall, M. Foss-Feig, and A. V. Gorshkov, Phys. Rev. B 93, 041102 (2016).

100 L. Lepori and L. Dell'Anna, New Journal of Physics 19, 103030 (2017)

101 K. Patrick, T. Neupert, and J. K. Pachos, Phys. Rev. Lett. 118, 267002 (2017)

102 O. Viyuela, L. Fu, and M. A. Martin-Delgado, Phys. Rev. Lett. 120, 017001 (2018)

103 L. Lepori, D. Giuliano, and S. Paganelli, Phys. Rev. B 97, 041109 (2018)

104 D. Vodola, L. Lepori, E. Ercolessi, A. V. Gorshkov, and G. Pupillo, Phys. Rev. Lett. 113, 156402 (2014).

105 F. Ares, J. G. Esteve, F. Falceto, and A. R. de Queiroz, Phys. Rev. A 92, 042334 (2015)

106 Z.-X. Gong, M. Foss-Feig, F. G. S. L. Brandão, and A. V. Gorshkov, Phys. Rev. Lett. 119, 050501 (2017)

107 N. Roy, A. Sharma, and R. Mukherjee, arXiv:1812.08938 (2018)

108 L. S. Levitov, Europhysics Letters (EPL) 9, 83 (1989)

109 L. S. Levitov, Phys. Rev. Lett. 64, 547 (1990)

110 A. D. Mirlin, Y. V. Fyodorov, F.-M. Dittes, J. Quezada, and T. H. Seligman, Phys. Rev. E 54, 3221 (1996)

111 A. Rodríguez, V. A. Malyshev, and F. Domínguez-Adame, J. Phys. A 33, L161 (2000)

112 A. Rodríguez, V. A. Malyshev, G. Sierra, M. A. Martín-Delgado, J. Rodríguez-Laguna, and F. Domínguez-Adame, Phys. Rev. Lett. 90, 027404 (2003)
113 F. A. B. F. de Moura, A. V. Malyshev, M. L. Lyra, V. A. Malyshev, and F. Domínguez-Adame, Phys. Rev. B 71, 174203 (2005)

114 G. L. Celardo, R. Kaiser, and F. Borgonovi, Phys. Rev. B 94, $144206(2016)$

115 X. Deng, V. E. Kravtsov, G. V. Shlyapnikov, and L. Santos, Phys. Rev. Lett. 120, 110602 (2018)

116 D. Vodola, L. Lepori, E. Ercolessi, and G. Pupillo, New J. Phys. 18, 015001 (2016)

117 T. Koffel, M. Lewenstein, and L. Tagliacozzo, Phys. Rev. Lett. 109, 267203 (2012)

118 A. L. Burin, Phys. Rev. B 91, 094202 (2015)

119 E. Lieb, T. Schultz, and D. Mattis, Ann. Phys. (N.Y.) 16, 407 (1961)

${ }^{120}$ P. Hauke and M. Heyl, Phys. Rev. B 92, 134204 (2015)

121 H. Li, J. Wang, X.-J. Liu, and H. Hu, Phys. Rev. A 94, 063625 (2016)

122 A. O. Maksymov, N. Rahman, E. Kapit, and A. L. Burin, Phys. Rev. A 96, 057601 (2017)

123 C. L. Baldwin, C. R. Laumann, A. Pal, and A. Scardicchio, Phys. Rev. Lett. 118, 127201 (2017)

${ }^{124}$ D. A. Abanin and Z. Papić, Annalen der Physik 529, 1700169 (2017)

125 L. Lepori, D. Vodola, G. Pupillo, G. Gori, and A. Trombettoni, Ann. Phys. 374, 35 (2016)

126 M. F. Maghrebi, Z.-X. Gong, M. Foss-Feig, and A. V. Gorshkov, Phys. Rev. B 93, 125128 (2016)

127 L. Lepori, A. Trombettoni, and D. Vodola, Journal of Statistical Mechanics: Theory and Experiment 2017, 033102 (2017).

128 S. R. White, Phys. Rev. Lett. 69, 2863 (1992)

129 http://itensor.org/

130 J. J. Sakurai and J. Napolitano, Modern Quantum Mechanics, 2nd ed. (Addison-Wesley, 2011).

131 A. Altland and B. Simons, Condensed Matter Field Theory (Cambridge University Press, Cambridge, England, 2006).

132 H. Bruus and K. Flensberg, Many-Body Quantum Theory in Condensed Matter Physics: An Introduction (Oxford University Press, Oxford, 2004).

133 M. Abramowitz and I. A. Stegun, Handbook of Mathematical Functions (Dover, 1964).

134 F. W. J. Olver, D. W. Lozier, R. F. Boisvert, and C. W. Clark, NIST Handbook of Mathematical Functions (Cambridge University Press, Cambridge, England, 2010).

135 K. Gottfried and T.-M. Yan, Quantum Mechanics: Fundamentals (Springer-Verlag, 2003). 